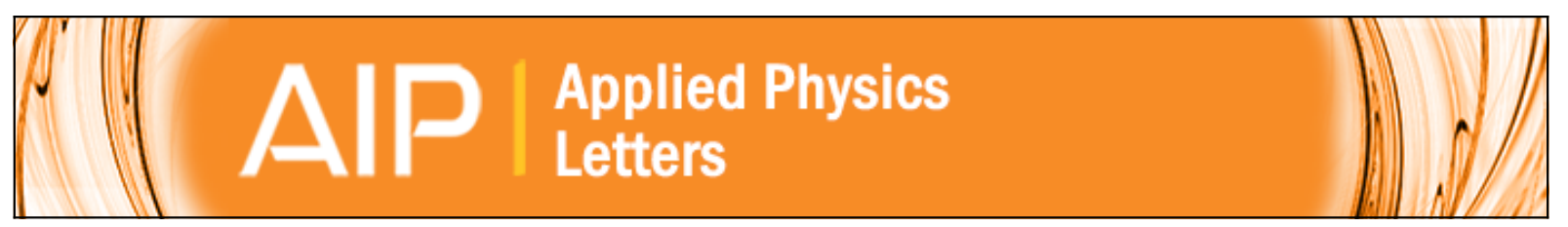

\title{
Mechanism of aluminium bio-mineralization in the apoferritin cavity
}

M. Chiarpotto, G. Ciasca, M. Vassalli, C. Rossi, G. Campi, A. Ricci, B. Bocca, A. Pino, A. Alimonti, P. De Sole, and M. Papi

Citation: Applied Physics Letters 103, 083701 (2013); doi: 10.1063/1.4818749

View online: http://dx.doi.org/10.1063/1.4818749

View Table of Contents: http://scitation.aip.org/content/aip/journal/apl/103/8?ver=pdfcov

Published by the AIP Publishing

\section{Articles you may be interested in}

Non-thermal mechanism of weak microwave fields influence on neurons

J. Appl. Phys. 114, 104701 (2013); 10.1063/1.4821027

Size-dependent structural evolution of the biomineralized iron-core nanoparticles in ferritins Appl. Phys. Lett. 102, 133703 (2013); 10.1063/1.4801310

Entropic potential field formed for a linear-motor protein near a filament: Statistical-mechanical analyses using simple models

J. Chem. Phys. 133, 045103 (2010); 10.1063/1.3462279

Effect of osteogenesis imperfecta mutations in tropocollagen molecule on strength of biomimetic tropocollagenhydroxyapatite nanocomposites

Appl. Phys. Lett. 96, 023703 (2010); 10.1063/1.3279158

Theory of competitive adsorption-nucleation in polypeptide-mediated biomineralization

J. Chem. Phys. 130, 161101 (2009); 10.1063/1.3126582

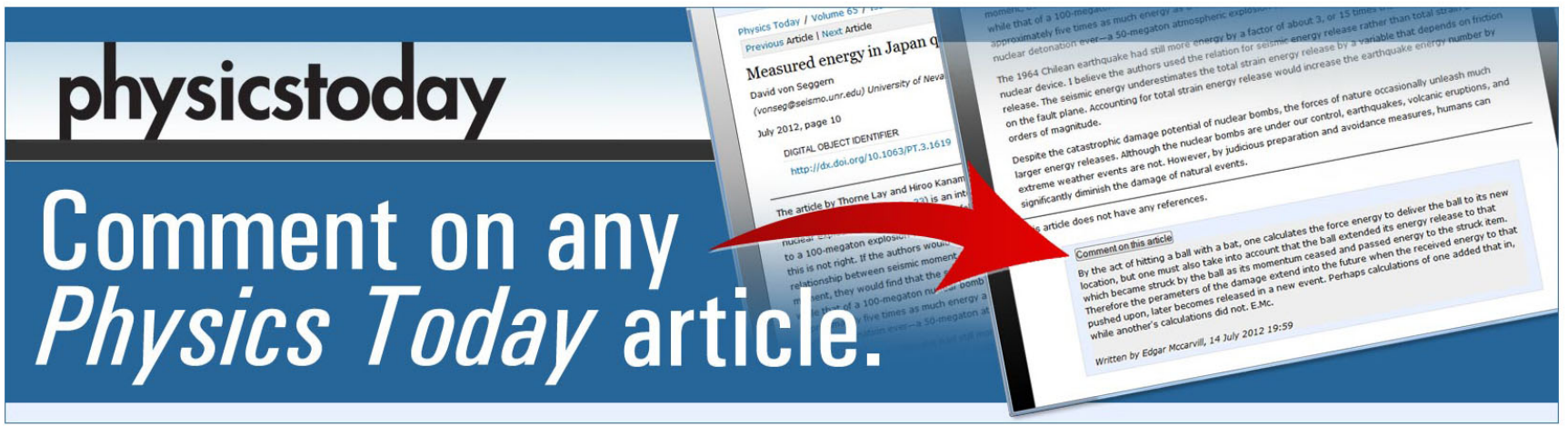




\title{
Mechanism of aluminium bio-mineralization in the apoferritin cavity
}

\author{
M. Chiarpotto, ${ }^{1, a)}$ G. Ciasca, ${ }^{1, a)}$ M. Vassalli, ${ }^{2}$ C. Rossi, ${ }^{3}$ G. Campi, ${ }^{4}$ A. Ricci, ${ }^{5}$ B. Bocca, ${ }^{6}$ \\ A. Pino, ${ }^{6}$ A. Alimonti, ${ }^{6}$ P. De Sole, ${ }^{3}$ and M. Papi ${ }^{1, b)}$ \\ ${ }_{1}^{1}$ Physics Institute, Catholic University, Largo Francesco Vito 1, 8, 00168 Rome, Italy \\ ${ }^{2}$ Institute of Biophysics, National Research Council, Via De Marini, 6 Genoa, Italy \\ ${ }^{3}$ Department of Clinical Biochemistry, Catholic University, Largo Francesco Vito 1, 8, 00168 Rome, Italy \\ ${ }^{4}$ Institute of Crystallography, CNR, Via Salaria Km 29.300, 00016 Monterotondo, Rome, Italy \\ ${ }^{5}$ Deutsches Elektronen-Synchrotron DESY, Notkestr. 85, D-22607 Hamburg, Germany \\ ${ }^{6}$ Applied Toxicology Laboratory, National Health Institute, Viale Regina Elena 299, 00161 Rome, Italy
}

(Received 19 November 2012; accepted 22 July 2013; published online 20 August 2013)

\begin{abstract}
Many experimental evidences point out the correlation between the presence of aluminum-ferritin complex and neursopathological disorders. In these complexes, two different ranges of Aluminium (Al) atoms are usually found, i.e., just few atoms or several hundreds. Here, we investigated the invitro $\mathrm{Al}$-apoferritin binding, with the aim to elucidate the mechanism behind the formation of $\mathrm{Al}$ ferritin complexes in-vivo. To this purpose, we studied the mineralization of $\mathrm{Al}$ in its ionic and complexed form with citrate demonstrating that high Al levels found in clinical studies can be obtained only conveying Al by small physiological ligands. (C) 2013 AIP Publishing LLC .
\end{abstract}

[http://dx.doi.org/10.1063/1.4818749]

Ferritin, the major cellular iron-storage protein, contains up to 4500 iron $(\mathrm{Fe})$ atoms per molecule, mainly as crystalline ferrihydrite. ${ }^{1}$ Although ferritin has its main role in iron storage, several evidences suggest that it functions as a general metal detoxicant, binding in-vivo metals other than iron, such as aluminium $(\mathrm{Al}),{ }^{2-7}$ which has been recognized as a contributing factor in several neurological disorders. ${ }^{8}$

The frequent isolation of $\mathrm{Al}$-ferritin complexes, especially from Alzheimer Disease (AD) patients, ${ }^{3,5,9,10}$ contributes to strengthen the suggested role for ferritin in metal toxicity. The observed increase of $\mathrm{Al}$ bound to brain ferritin has been indeed proposed as a detoxification mechanism which protects the brain metabolism from $\mathrm{Al}$ that cannot be cleared by other mechanisms.

By the way, the mechanism behind the $\mathrm{Al}$ complex formation in vivo is still controversial also because two different ranges of $\mathrm{Al}$ bound to ferritin have been generally found: few atoms in some studies ${ }^{5,6}$ and hundreds or even thousands in others. ${ }^{3,4,9}$ Furthermore in this latter case also a very high $\mathrm{Al} / \mathrm{Fe}$ molar ratio has been observed (in some specific case up to 6). ${ }^{4,9}$ In this work, we investigate in-vitro by Sector Field Inductive Coupled Mass Spectroscopy (SF-ICP-MS) and Small-Angle $\mathrm{X}$ ray Scattering (SAXS) ${ }^{11}$ the binding of $\mathrm{Al}$ to apoferritin with the aim to provide a better understanding of the mechanism underlying these two different uptakes of aluminum. In biological systems, aluminum can be found in its ionic form or complexed with small ligands which increase its solubility. Therefore, we focus on both cases, ionic $\mathrm{Al}$ and $\mathrm{Al}$ complexed with citrate, the most likely $\mathrm{Al}$ ligand in blood. ${ }^{12}$

Incubation experiments with the ionic form of aluminium have been carried out using horse spleen apoferritin (ApoFt) purchased from Sigma. The mineralization process was induced by incubating apoferritin $0.3 \mu \mathrm{M}$ for $48 \mathrm{~h}$ in eight

\footnotetext{
${ }^{\text {a) }}$ M. Chiarpotto and G. Ciasca contributed equally to this work.

${ }^{b)}$ Author to whom correspondence should be addressed. Electronic mail: m.papi@rm.unicatt.it
}

tris(hydroxymethyl)aminomethane (TRIS) buffered reaction solutions containing different iron salt $\left(\mathrm{FeSO}_{4} \cdot 7 \mathrm{H}_{2} \mathrm{O}\right)$ and aluminium salt $\left(\mathrm{AlK}\left(\mathrm{SO}_{4}\right)_{2} \bullet 12 \mathrm{H}_{2} \mathrm{O}\right)$ concentrations. The composition of the incubated solutions in term of aluminum and iron is summarized in Table I, where the iron/apoferritin $(\mathrm{Fe} / \mathrm{ApoFt})$ molar ratio is also reported. One can observe that $\mathrm{Al}$ concentration in the reactions solution decreases when iron concentration increases. This choice is aimed to stress the role of iron in the mineralization process of aluminum. After incubation, apoferritin has been separated from the reaction solution using centrifugal concentrators produced by Sartorius (product number VS0612, mass cut off $5 \mathrm{kDa}$, protein recovery $>96 \%$ ). Thus, the protein has been diluted in a TRIS buffer solution at the same $\mathrm{pH}$. Several centrifugation and dilution cycles have been performed to completely eliminate residual $\mathrm{Al}$ and $\mathrm{Fe}$ content in the apoferritin solution (i.e., $\mathrm{Al}$ and $\mathrm{Fe}$ not bonded to apoferritin).

The ferritin mineral core reconstitution was verified by Small Angle X-rays Scattering (SAXS). SAXS measurements were carried out on the Austro-SAXS beamline at the Synchrotron Radiation Facility ELETTRA (Trieste, Italy). ${ }^{13}$ Samples have been further concentrated and measured in a $1.5 \mathrm{~mm}$-diameter capillary sample holder, using an incident $\mathrm{X}$-ray energy of $8 \mathrm{keV}$. Data acquisition has been performed with an image plate detector Mar 345 in a s range $0.01-5 \mathrm{~nm}^{-1}$. To allow for accurate subtraction of the background scattering, solvent scattering was acquired before and after measuring the protein solutions. Each acquisition was averaged over $500 \mathrm{~s}$ to optimize signal to noise ratio and avoid sample damage. The scattered intensity I(s) as a function of the X-ray-exchanged momentum $\mathrm{s}$ has been processed by the indirect transform program GIFT $^{14}$ to evaluate the pair distribution function (PDF) and the corresponding electron density profile.

In Fig. 1 (panels (a)-(e)), we show the I(s) scattering curves of the apoferritin samples incubated in the presence of different $\mathrm{Fe} / \mathrm{ApoFt}$ molar ratios (Table I). 
TABLE I. Composition of the incubated solutions in terms of ionic aluminum and iron.

\begin{tabular}{lcccr}
\hline \hline $\mathrm{pH}^{\mathrm{a}}$ & {$[\mathrm{Fe}] \mu \mathrm{M}^{\mathrm{b}}$} & {$[\mathrm{Al}] \mu \mathrm{M}^{\mathrm{c}}$} & {$[\mathrm{ApoFt}] \mu \mathrm{M}^{\mathrm{d}}$} & $\mathrm{Fe} / \mathrm{ApoFt}^{\mathrm{e}}$ \\
\hline 7.4 & 0 & $15 \pm 3$ & 0.3 & 0 \\
7.4 & $75 \pm 15$ & $12.5 \pm 2.5$ & 0.3 & 250 \\
7.4 & $150 \pm 30$ & $10 \pm 2$ & 0.3 & 500 \\
7.4 & $225 \pm 40$ & $7.5 \pm 1.5$ & 0.3 & 750 \\
7.4 & $300 \pm 50$ & $5 \pm 1$ & 0.3 & 1000 \\
7.4 & $375 \pm 70$ & $2.5 \pm 0.5$ & 0.3 & 1250 \\
7.4 & $450 \pm 80$ & 0 & 0.3 & 1500 \\
8.5 & 0 & $100 \pm 20$ & 0 & 0 \\
\hline \hline
\end{tabular}

${ }^{\mathrm{a}}$ The first column indicates the solution $\mathrm{pH}$.

${ }^{\mathrm{b}}[\mathrm{Fe}] \mu \mathrm{M}$ is the iron concentration in the incubation solutions expressed in micromolar units.

${ }^{\mathrm{c}}[\mathrm{Al}] \mu \mathrm{M}$ is the ionic aluminium concentration in the incubation solutions expressed in micromolar units.

${ }^{\mathrm{d}}[\mathrm{ApoFt}] \mu \mathrm{M}$ is the apoferritin concentration in the reaction solution expressed in micromolar units.

${ }^{\mathrm{e}} \mathrm{Fe} / \mathrm{ApoFt}$ is the iron/apoferritin molar ratio in the incubation solutions.
Only selected curves have been shown for clarity, namely, Fe/ApoFt $=1500$ (panel (a)), 1250 (panel (b)), 1000 (panel (c)), 750 (panel (d)), and 250 (panel (e)). In Fig. 1 (panels (f)-(l)), we report the corresponding PDF curves obtained by using the indirect transformation technique implemented in the GIFT software package. A gradual shift of the PDF peak position between $4 \mathrm{~nm}$ and $8 \mathrm{~nm}$ can be clearly observed: the higher the $\mathrm{Fe} / \mathrm{ApoFt}$ molar ratio, the lower the PDF peak position. This behavior indicates that the apoferritin cavity undergoes a continuous transition between an empty state and a filled state, ${ }^{15-17}$ demonstrating that the incubation procedure induces a re-mineralization of the apoferritin cavity. To gain a better knowledge about the internal structure of the incubated apoferritin samples, the PDF curves were deconvoluted by using the DECON software with the aim to recover the radial density distribution profile. Polidispersity has been also taken into account by using a Shifted Schulz distribution. The optimal value for the degree of polidispersity was found between $15 \%$ and $20 \%$ for all samples. ${ }^{14}$ Fig. $1(\mathrm{~m})$ shows the radial density distribution profiles $\rho(\mathrm{r})$ of the incubated apoferritin. Two regions have (a)

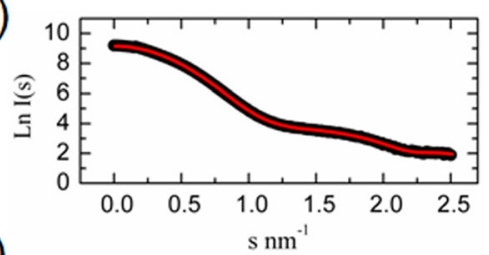

(b)

(c)

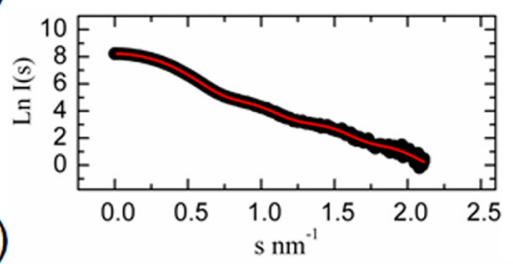

(d)
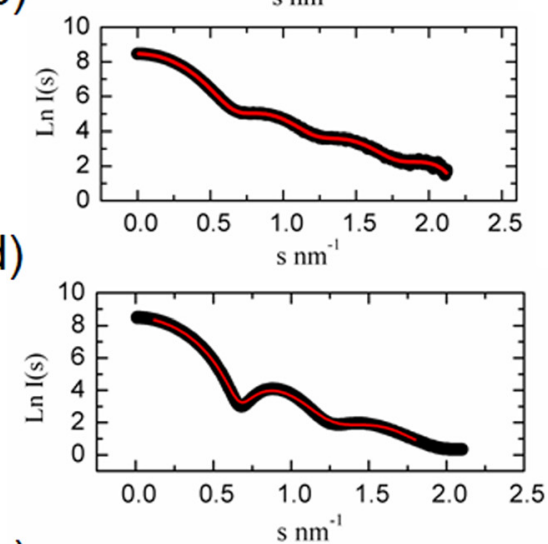

(e)

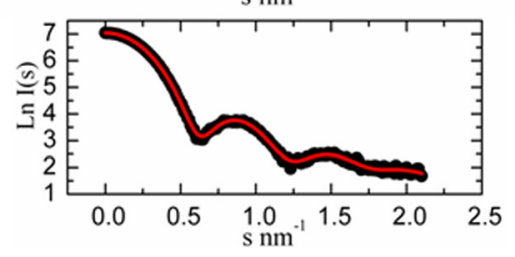

(f)

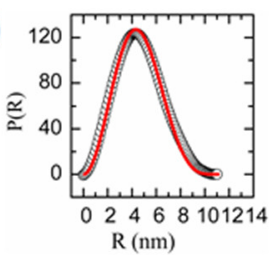

(g)

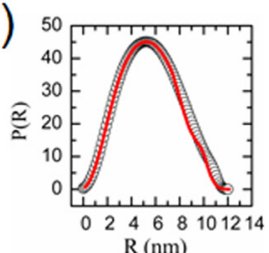

(h)

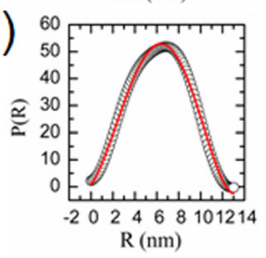

(i)

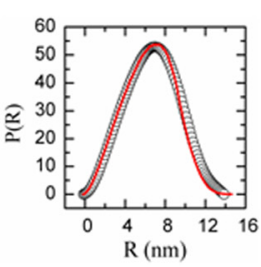

(I)

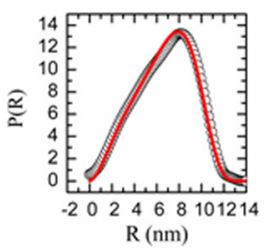

(m)

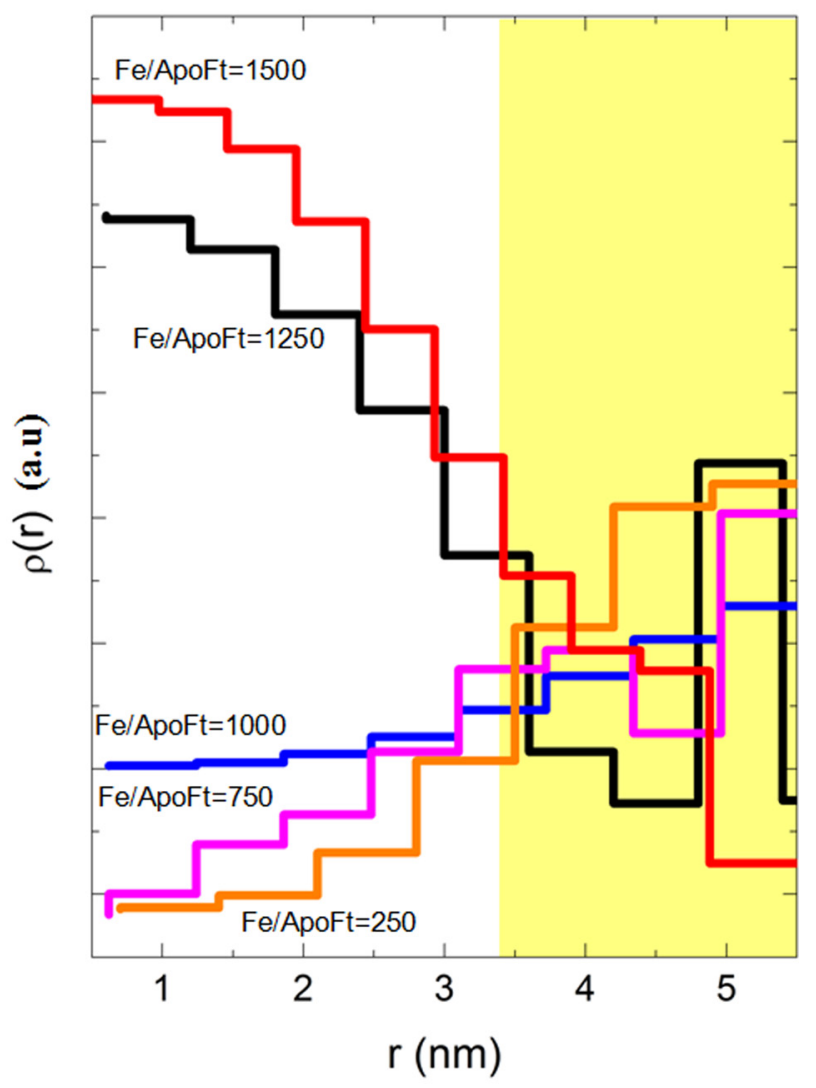

FIG. 1. Panels (a)-(e): Scattering curves of the apoferritin samples incubated in the presence of different Fe/ApoFt molar ratios, namely, 1500 (panel (a)), 1250 (panel (b)), 1000 (panel (c)), 750 (panel (d)), and 250 (panel (e)). The experimental data are shown as filled dots. The fits of the experimental data, performed with the software GIFT, are shown as red continuous lines. Panels (f)-(l): Pair distribution functions obtained by indirect Fourier Transform of the scattering curves using the software package GIFT (open dots). Fits of the pair distribution function obtained by the software DECON (continuous red lines). Panels are presented in order of decreasing Fe/ApoFt molar ratio. Panel (m): Density distribution profiles obtained by the software DECON fitting the pair distribution function. The values of the radius $r$ corresponding to the protein shell are highlighted in yellow $(r>3.4 \mathrm{~nm})$. 
been highlighted, depending on the radial coordinate $r$ : the first region $(\mathrm{r}<3.4 \mathrm{~nm})$ corresponds to the apoferritin cavity, the second $(r>3.4 \mathrm{~nm})$ to the protein shell. As suggested by the behavior of the PDF curves, the density distribution in the first region increases with the increasing of the Fe/ApoFt molar ratio, revealing the occurrence of a mineralization process. One can also observe that, for $\mathrm{Fe} / \mathrm{ApoFt}=250$, the centre of the apoferritin cavity $(\mathrm{r}<2 \mathrm{~nm})$ appears almost empty, confirming that the ferritin mineralization process starts form the outer part of the cavity. ${ }^{17}$

Metal ion content ( $\mathrm{Al}, \mathrm{Fe}$ ) bonded to the apoferritin molecule after incubation was measured by SF-ICP-MS, using the ELEMENT 2 model from Thermo Fisher (Bremen, Germany) in the medium resolution mode $(\mathrm{m} / \Delta \mathrm{m}=4000)$. The isotopes chosen for quantification were ${ }^{27} \mathrm{Al}$ for aluminium and ${ }^{56} \mathrm{Fe}$ for iron. The medium resolution approach allowed us to separate the analytical peaks from the interfering ones (i.e., ${ }^{13} \mathrm{C}^{14} \mathrm{~N},{ }^{12} \mathrm{C}^{15} \mathrm{~N},{ }^{54} \mathrm{Fe}++$, and ${ }^{11} \mathrm{~B}^{16} \mathrm{O}$ on mass 27; and ${ }^{40} \mathrm{Ar}^{16} \mathrm{O}$ and ${ }^{40} \mathrm{Ca}^{16} \mathrm{O}$ on mass 56 ). The number of $\mathrm{Al}$ and $\mathrm{Fe}$ atoms bound to apoferritin was calculated by dividing the $\mathrm{Al}$ and Fe concentration measured by SF-ICP-MS by the apoferritin concentration, as described in Ref. 17.

In Fig. 2(a), we show the number of Fe atoms bound to ferritin versus the Fe concentration in the reaction solution. A linear trend can be observed, confirming the occurrence of the mineralization process, as shown by SAXS measurements.

In Fig. 2(b), we show the number of $\mathrm{Al}$ atoms bound to ferritin-measured on the same samples-as a function of the $\mathrm{Al}$ concentration in the reaction solution (lower $\mathrm{x}$-axis) and the iron concentration in the reaction solution (upper $\mathrm{x}$-axis). One might observe that $\mathrm{Al}$ concentration is significantly lower than Fe concentration: it depends on the fact that $\mathrm{Al}$ solubility is strongly decreased by the precipitation of $\mathrm{Al}(\mathrm{OH})_{3}$ at neutral $\mathrm{pH}$. At this $\mathrm{pH}, \mathrm{Al}$ is present mainly as the hydrated specie $\mathrm{Al}(\mathrm{OH})_{4}{ }^{-}$, which is considered the physiological form of aluminium in solution. ${ }^{11}$ In Fig. 2(b), two regions can be distinguished, depending on $\mathrm{Al}$ concentration in the reaction solution. Below $[\mathrm{Al}]=13 \mu \mathrm{M}$, the incubation has been carried out in the presence of iron. Above, the $\mathrm{Al}$ incubation has been performed in the absence of iron salt in the reaction solution.

In the first region, the number of $\mathrm{Al}$ atoms increases linearly with the Al concentration in solution, irrespectively of the iron concentration, which-in its turn-decreases as the $\mathrm{Al}$ one increases. The observed linear increase of $\mathrm{Al}$ in this region of the plot strongly confirms that the main limiting factor for Al uptake during the iron core reconstruction is the Al concentration in solution, as proposed in Ref. 3 .

Conversely, at $[\mathrm{Al}]=15 \mu \mathrm{M}$ and $[\mathrm{Al}]=100 \mu \mathrm{M}$, the number of $\mathrm{Al}$ atoms bound to ferritin decreases abruptly. It is worth stressing here that concentrations higher than $15 \mu \mathrm{M}$ were obtained by raising the solution $\mathrm{pH}$ up to 8.5. At this $\mathrm{pH}, \mathrm{Al}$ solubility increases of about one order of magnitude and-at the same time-ferritin does not undergo dramatic modification. Moreover, for even higher $\mathrm{pH}$, ferritin has been demonstrated to mineralize both $\mathrm{Fe}$ and $\mathrm{Al}$ atoms depending on their concentration in the reaction solution. ${ }^{17}$ The abrupt decrease of the $\mathrm{Al}$ content in this region indicates that the binding of ionic Al occurs during the iron core formation, whereas, in the absence of iron, the Al-ferritin complex is not formed. Even if $\mathrm{Al}$ absorption on the protein surface cannot be completely excluded, our data suggest that this phenomenon contributes to a limited extent to the $\mathrm{Al}$ uptake. It is well known that the formation of iron core in ferritin involves the binding and the oxidation of ferrous iron $\left(\mathrm{Fe}^{2+}\right)$ to ferric iron $\left(\mathrm{Fe}^{3+}\right)$, but a similar mechanism cannot be applied to aluminum in its ionic form, since the +2 oxidation state is not allowed. However, once a small core of ferrihydrite is formed inside the ferritin molecule, auto oxidation of iron occurs on the surface of the crystalline core independently of the protein subunits, ${ }^{18}$ implying that metals other than iron can be mineralized in the ferritin core following mechanisms that occurs also in minerals. Having in mind this consideration, our findings suggest the occurrence of two possible mechanisms for the formation of Al-ferritin complexes in vivo in the presence of ionic Al which are, both, common phenomena in the mineralization pathway of natural and synthetic ferrihydrite-i.e., specific $\mathrm{Al}^{3+}$ substitution in $\mathrm{Fe}^{3+}$ (Hydro)oxide or unspecific $\mathrm{Al}$ absorption in the mineral core, during the iron core formation. ${ }^{19}$ In the latter case, Al would absorb on the surface of a growing core, being trapped by the growing core itself. Recent quantum mechanical calculations reveal indeed that the substitution of Al into ferrihydrite nanoparticles has a relatively small effect on the structural parameters, such as $\mathrm{Fe}-\mathrm{O}$ and $\mathrm{Fe}-\mathrm{Fe}$ distances and angles. In particular, $\mathrm{Al}(\mathrm{OH})_{4}{ }^{-}$substitution into (a)

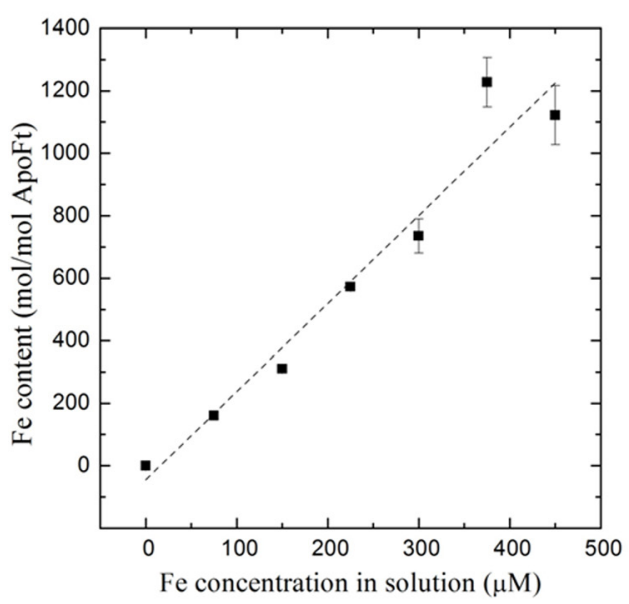

(b)

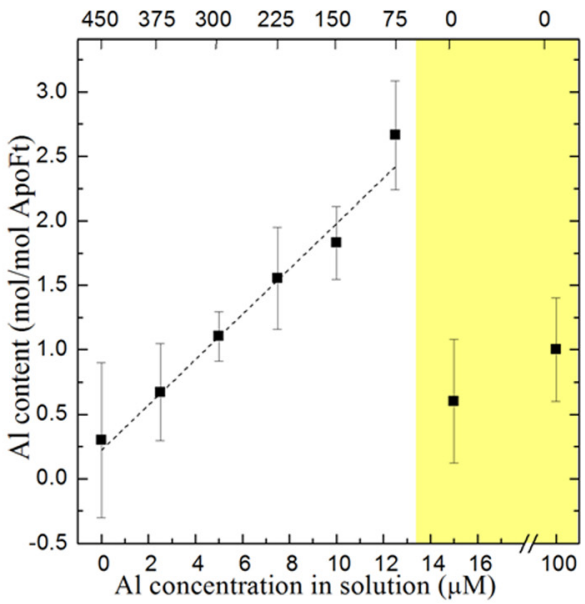

FIG. 2. Panel (a): Number of mineralized $\mathrm{Fe}$ atoms within the ferritin cavity as a function of the iron concentration in the incubation solution. Panel (b): Number of mineralized $\mathrm{Al}$ atoms within the ferritin cavity as a function of the $\mathrm{Al}$ concentration in the incubation solution. 
TABLE II. Composition of the incubated solutions in term of complexed aluminum and iron.

\begin{tabular}{|c|c|c|c|c|c|c|c|}
\hline $\mathrm{pH}^{\mathrm{a}}$ & {$[\mathrm{Fe}] \mathrm{mM}^{\mathrm{b}}$} & {$[\mathrm{Al}] \mathrm{mM}^{\mathrm{c}}$} & {$[\mathrm{ApoFt}] \mu \mathrm{M}^{\mathrm{d}}$} & $\mathrm{Fe} / \mathrm{ApoFt}^{\mathrm{e}}$ & $\mathrm{Al} / \mathrm{ApoFt}{ }^{\mathrm{f}}$ & Kpcs (a.u. $)^{g}$ & $\mathrm{R}_{\mathrm{H}}(\mathrm{nm})^{\mathrm{h}}$ \\
\hline 7.4 & 0.45 & 0.125 & 0.3 & $200 \pm 10$ & $74 \pm 9$ & $139 \pm 12$ & $6.09 \pm 1.3$ \\
\hline 7.4 & 0.45 & 0.25 & 0.3 & $230 \pm 15$ & $170 \pm 13$ & $150 \pm 12$ & $6.20 \pm 1.5$ \\
\hline 7.4 & 0.45 & 0.5 & 0.3 & $200 \pm 15$ & $280 \pm 17$ & $162 \pm 13$ & $6.52 \pm 1.6$ \\
\hline 7.4 & 0.45 & 1 & 0.3 & $230 \pm 15$ & $610 \pm 25$ & $202 \pm 14$ & $6.44 \pm 1.4$ \\
\hline 7.4 & 0.45 & 2 & 0.3 & $194 \pm 14$ & $1235 \pm 35$ & $312 \pm 17$ & $5.27 \pm 1.4$ \\
\hline 7.4 & 0 & 0.125 & 0.3 & 0 & $85 \pm 9$ & $159 \pm 12$ & $6.40 \pm 1.5$ \\
\hline 7.4 & 0 & 2 & 0.3 & 0 & $2000 \pm 45$ & $371 \pm 19$ & $5.67 \pm 1.4$ \\
\hline
\end{tabular}

${ }^{\mathrm{a}}$ The first column indicates the solution $\mathrm{pH}$.

${ }^{\mathrm{b}}[\mathrm{Fe}] \mathrm{mM}$ is the iron concentration in the incubation solutions expressed in millimolar units.

${ }^{\mathrm{c}}$ [Al] $\mathrm{mM}$ is the Al-citrate concentration in the incubation solutions expressed in millimolar units.

${ }^{\mathrm{d}}[\mathrm{ApoFt}] \mu \mathrm{M}$ is the apoferritin concentration in the reaction solution expressed in micromolar units.

${ }^{\mathrm{e}} \mathrm{Fe} / \mathrm{ApoFt}$ is the iron/apoferritin molar ratio in the incubation solutions.

${ }^{\mathrm{f}} \mathrm{Al} / \mathrm{ApoFt}$ is the aluminium/apoferritin molar ratio in the incubation solutions.

${ }^{\mathrm{g}} \mathrm{Kpcs}$ (a.u.) is the average scattering intensity of the incubated apoferritin expressed in arbitrary units.

${ }^{h} R_{H}(n m)$ is the hydrodynamic radius of the incubated apoferritin expressed in nanometers.

ferrihydrite has a Gibbs free energy ranging from 50 to $100 \mathrm{~kJ} / \mathrm{mol}$ depending on the model used to perform calculations, whereas $\mathrm{Al}^{3+}$ substitution ranges between about -20 and $20 \mathrm{~kJ} / \mathrm{mol}^{20}$

As mentioned above, $\mathrm{Al}$ can be found in-vivo not only in its ionic form but also in complexed form. To account for the latter, we incubated apoferritin $0.3 \mu \mathrm{M}$ in the presence of different concentrations of Al-citrate ranging from $0.125 \mathrm{mM}$ to $2 \mathrm{mM}$ and in the presence of Fe $0.45 \mathrm{mM}$. Alcitrate has been prepared in acid environment $(\mathrm{pH}=3)$ according to Dedman and coworkers ${ }^{6}$ and subsequently dissolved at physiological $\mathrm{pH}$ in the presence of empty apoferritin $0.3 \mu \mathrm{M}$ and $\mathrm{Fe} 0.45 \mathrm{mM}$. The apoferritin incubation was also performed in the absence of Fe for two Al-citrate concentrations, namely, $0.125 \mathrm{mM}$ and $2 \mathrm{mM}$, in order to elucidate the role of iron in the mineralization process. $\mathrm{Al}$ and $\mathrm{Fe}$ apoferritin content after incubation was determined by SF-ICP-MS, using the same procedure as described above. The composition of the incubated solutions is summarized in Table II.
Fig. 3(a) shows the number of $\mathrm{Al}$ atoms mineralized in the ferritin cavity as a function of the Al-citrate concentration in the reaction solution in the presence of $\mathrm{Fe}$ (open black dots) and in the absence of iron (filled black dots). The number of mineralized $\mathrm{Fe}$ atoms is also shown (red filled circles).

Our SF-ICP-MS measurements show a constant amount of about $200 \mathrm{Fe}$ atoms per apoferritin molecule, independently of the Al-citrate concentration. Such an amount is significantly lower than that measured in the previous experiment for the same iron concentration in the reaction solution. Conversely, a similar amount of $\mathrm{Fe}$ atoms was measured for a Fe concentration of $0.1 \mathrm{mM}$.

The number of mineralized $\mathrm{Al}$ atoms per apoferritin molecule increases linearly with the Al-citrate concentration in the reaction solution up to an $\mathrm{Al}$ content of about 1250 atoms per apoferritin molecule. Such an elevated $\mathrm{Al}$ amount is similar to that recently found in-vivo in the serum ferritin extracted from AD patients. ${ }^{9}$ In contrast to the results shown in Fig. 2(b), an even higher amount of $\mathrm{Al}$ atoms (about 2000 (a)

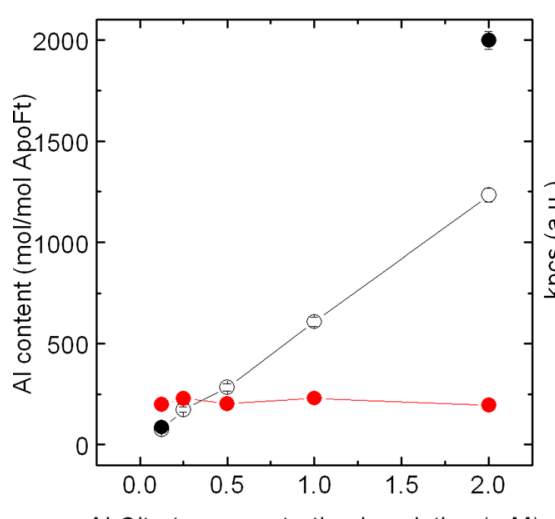

Al-Citrate concentration in solution ( $\mathrm{mM}$ ) (b)

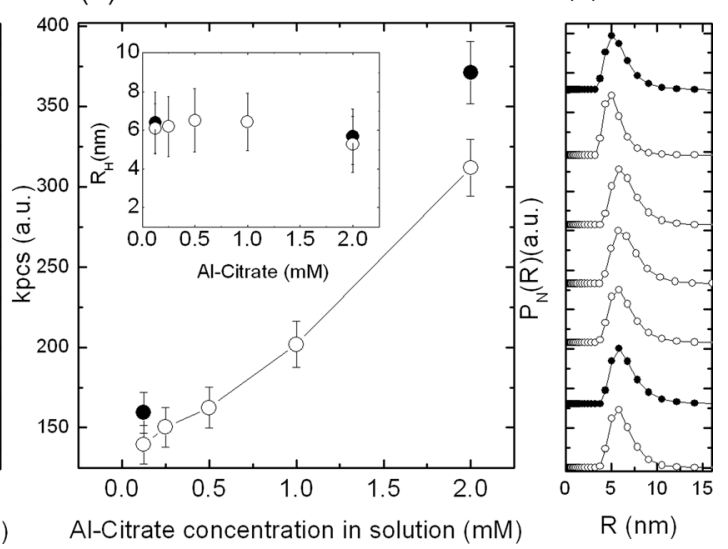

FIG. 3. Panel (a): Number of $\mathrm{Al}$ atoms mineralized in the ferritin cavity as a function of the Al-citrate concentration in the reaction solution in the presence of $\mathrm{Fe}$ (open black dots) and in the absence of iron (filled black dots). The number of mineralized Fe atoms is also shown (red filled circles). Apoferritin in solution was $0.3 \mu \mathrm{M}$. Panel (b): Average scattered intensity of the incubated apoferritin sample as a function of the Al-citrate concentration in the reaction solution. Inset of panel (b): Average hydrodynamic radius versus Al-citrate concentration in the reaction solution in presence (open black dots) and absence (filled black dots) of iron. Panel (c): Al-citrate loaded apoferritin number distribution in presence (open black dots) and absence (filled black dots) of iron. Panels are presented in order of decreasing Al/ApoFt molar ratio; the values are shown in Table II. 
$\mathrm{Al}$ atoms per apoferritin molecule) is mineralized in the apoferritin cavity in the absence of iron.

The occurrence of a mineralization process within the ferritin was also confirmed by dynamic light scattering (DLS) measurements performed by using a commercial instrument (ZetasizerNanoZS, Malvern, United Kingdom). Fig. 3(b) shows the average scattering intensity of the incubated apoferritin in the presence (open dots) and in the absence (filled dots) of iron. One can notice that, as expected, the integrated scattering intensity increases with increasing the Al-citrate concentration in solution. On the other hand, the hydrodynamic radius $\mathrm{R}_{\mathrm{H}}$ of the incubated apoferritin (inset of Fig. 3(b)), obtained by the average of the DLS number distribution $\mathrm{P}_{\mathrm{N}}\left(\mathrm{R}_{\mathrm{H}}\right)$ shown in Fig. 3(c), remains constant within the experimental error. It is well know that an increase in the average scattering intensity is associated with an increase in the molecular mass and/or in the scattering contrast of the scattering objects. This result, coupled to the constancy of $R_{H}$, is consistent with the occurrence of a re-mineralization process within the apoferritin cavity.

On the whole, our uptake experiments show a very different outcome between ionic $\mathrm{Al}$ and $\mathrm{Al}$-citrate.

In a clinical study carried out on patients who died of $\mathrm{AD}$ and age matched controls, Fleming and co-workers reported an amount of about $20 \mathrm{Al}$ atoms per brain ferritin in the case of AD's patients and about four $\mathrm{Al}$ atoms per brain ferritin in the case of age matched control. ${ }^{4}$ Subsequent studies carried on ferritin isolated from brain of renal dialysis patients showed an amount of $10 \mathrm{Al}$ atoms per ferritin molecule. ${ }^{5}$ Ward et al. reported a small amount of the radioactive tracer ${ }^{26} \mathrm{Al}$ associated with liver ferritin of $\mathrm{Al}$ loaded rat. ${ }^{7}$

The total $\mathrm{Al}$ amount per ferritin molecule found in the these studies is of the same order as the one shown in Fig. 2, suggesting that such a little amount of Al could derive, also in-vivo, from the mineralization of the ionic form of aluminum, mainly $\mathrm{Al}(\mathrm{OH})_{4}{ }^{-}$.

On the contrary, this incubation procedure fails to reproduce higher $\mathrm{Al}$ content found in other in-vivo studies ${ }^{3,9}$ probably because of the low solubility of $\mathrm{Al}$ in solution at neutral $\mathrm{pH}$.

On the other hand, $\mathrm{Al}$ in its complexed form shows a significantly higher uptake compared to its ionic form, reaching an amount of about $2000 \mathrm{Al}$ atoms/molecule. Interestingly, our incubation experiments with Al-citrate show an $\mathrm{Al} / \mathrm{Fe}$ molar ratio greater than 1 . Such a high value of $\mathrm{Al}$ and $\mathrm{Al} / \mathrm{Fe}$ molar ratio is comparable to those found in recent studies on clinical patients. ${ }^{4,9}$ These results suggest that this high amount of aluminum present in the apoferritin is likely conveyed by small ligands, such as citrate, which increases its solubility in physiological condition.
Concluding, it is worth to note that aluminium absorption and substitution have been found to induce structural modifications in iron oxide crystals, such as ferrihydrite, magnetite, hematite and goethite, influencing the nucleation of multiphase iron oxide minerals. ${ }^{19,20}$

Interestingly, recent studies reported the presence of different crystalline structures of normal brain ferritin when compared to pathological ferritin extracted from AD's patients. ${ }^{21,22}$ Therefore, it is likely that such modification of the multiphase ferritin core in pathological condition could be induced by the presence of $\mathrm{Al}$ atoms. This mechanism could have an important role in the pathological alterations typical of this and other neurodegenerative diseases as suggested in Ref. 9.

${ }^{1}$ P. M. Harrison and P. Arosio, Biochim. Biophys. Acta 1275, 161 (1996).

${ }^{2}$ T. Sakamoto, Y. Ogasawara, K. Ishii, H. Takahashi, and S. Tanabe, Neurosci. Lett. 366, 264 (2004).

${ }^{3}$ P. L. Spada, C. Rossi, A. Alimonti, B. Bocca, B. Ricerca, M. Bocci, M. Carvelli, C. Vulpio, G. Luciani, and P. De Sole, Clin. Biochem. 41, 997 (2008).

${ }^{4}$ J. T. Fleming and J. G. Joshi, Neurobiol. Aging 12(5), 413 (1991).

${ }^{5}$ D. J. Dedman, A. Treffry, J. M. Candy, G. A. Taylor, C. M. Morris, C. A. Bloxham, R. H. Perry, J. A. Edwardson, and P. M. Harrison, Biochem. J. 287, 509 (1992).

${ }^{6}$ D. J. Dedman, A. Treffry, and P. M. Harrison, Biochem. J. 287, 515 (1992).

${ }^{7}$ R. J. Ward, Y. Zhang, and R. R. Crichton, J. Inorg. Biochem. 87(1-2), 9 (2001).

${ }^{8}$ C. G. Fraga, P. I. Oteiza, M. S. Golub, M. E. Gershwin, and C. L. Keen, Toxicol. Lett. 51(2), 213 (1990).

${ }^{9}$ P. De Sole, C. Rossi, M. Chiarpotto, G. Ciasca, B. Bocca, A. Alimonti, A. Bizzarro, C. Rossi, and C. Masullo, Clin. Biochem. 46, 89 (2013).

${ }^{10}$ J. Joshi, S. Sczekan, and J. Fleming, Biol. Trace Elem. Res. 21, 105 (1989).

${ }^{11}$ H. Amenitsch, M. Rappolt, M. Kriechbaum, H. Mio, P. Laggner, and S. Bernstorff, J. Synchrotron Radiat. 5, 506 (1998).

${ }^{12}$ F. B. Martin, Clin. Chem. 32, 1797 (1986).

${ }^{13}$ H. Amenitsch, S. Bernstorff, M. Kriechbaum, D. Lombardo, H. Mio, M. Rappolt, and P. Laggner, J. Appl. Cryst. 30, 872 (1997).

${ }^{14}$ T. Frühwirth, G. Fritz, N. Freiberger, and O. Glatter, J. Appl. Cryst. 37, 703 (2004).

${ }^{15}$ N. Galvez, F. B. Fernandez, P. Sánchez, R. Cuesta, M. Ceolín, M. Clemente-León, S. Trasobares, M. López-Haro, J. J. Calvino, O. Stéphan, and J. M. Domínguez-Vera, J. Am. Chem. Soc. 130, 8062 (2008).

${ }^{16}$ G. Ciasca, M. Papi, M. Chiarpotto, M. Rodio, G. Campi, C. Rossi, P. De Sole, and A. Bianconi, Appl. Phys. Lett. 100, 073703 (2012).

${ }^{17}$ G. Ciasca, M. Chiarpotto, G. Campi, B. Bocca, M. Rodio, A. Pino, A. Ricci, N. Poccia, C. Rossi, A. Alimonti, H. Amenitsch, P. De Sole, and A. Bianconi, J. Nanopart. Res. 13, 6149 (2011).

${ }^{18}$ B. Xu and N. D. Chasteen, J. Biol. Chem. 266, 19965 (1991).

${ }^{19}$ C. M. Hansel, D. R. Learman, C. J. Lentini, and E. B. Ekstrom, Geochim.Cosmochim. Acta 75(16), 4653 (2011).

${ }^{20}$ J. D. Kubicki, M. Aryanpour, L. Kabalan, and Q. Zhu, Geoderma 189-190, 236 (2012).

${ }^{21}$ C. Quintana, J. M. Cowley, and C. Marhic, J. Struct. Biol. 147, 166 (2004).

${ }^{22}$ C. Quintana and L. Gutiérrez, Biochim. Biophys. Acta 1800(8), 770 (2010). 